\title{
A Survey on Estimation Schemes in Molecular Communications
}

\author{
Xinyu Huang, Yuting Fang, and Nan Yang
}

\begin{abstract}
This survey paper focuses on the estimation schemes in molecular communication (MC) systems. The existing studies in estimation schemes can be divided into parameter estimation (e.g., distance, diffusion coefficient, and flow velocity) and channel estimation. In this paper, we present, for the first time, a comprehensive survey on i) distance estimation, since distance is the most widely estimated parameter in current studies, ii) estimation of other parameters (i.e. the parameters excluding distance), and iii) channel estimation that focuses on the channel impulse response (CIR). Moreover, we examine the noise that may impact on the estimation performance and the metrics applied to evaluate the performance of different estimation schemes. Numerical results are provided to compare the performance of different distance estimation schemes. In addition, future research directions in parameter estimation and channel estimation are identified and discussed.
\end{abstract}

Index Terms-Molecular communication, parameter estimation, channel estimation, statistical model.

\section{INTRODUCTION}

Molecular communications (MC) is an emerging technology in the past decade, which has great potential to facilitate nanoscale communication. Instead of using electromagnetic waves as information carriers as in traditional wireless communication, MC uses small particles such as molecules or lipid vesicles to deliver information [1]. Moreover, $\mathrm{MC}$ owns unique merits such as biocompatibility and low energy consumption, which make MC more suitable for in vivo applications than other communication methods, e.g., electromagnetic methods.

Recently, some surveys and tutorials have discussed the benefits and challenges of MC from different perspectives, e.g., [1]-[5]. Specifically, [1] presented a detailed introduction on $\mathrm{MC}$ and provided an overall survey at the recent advances in the micro-scale MC and the macro-scale MC. In [2], the authors presented a survey on the applications of MC and molecular networks with the focus on targeted drug delivery. In [3], Jamali et al. provided a tutorial review on mathematical channel modeling for diffusive MC systems. In [4], the authors reviewed the contributions to the architectures of transmitter (TX) and receiver (RX) among nanomaterialbased nanomachines and/or biological entities and provided a complete overview of modulation, coding, and detection techniques employed for MC. Nakano et al. in [5] provided a comprehensive review on mobile MC. Although these studies

X. Huang and N. Yang are with the School of Engineering, Australian National University, Canberra, ACT 2600, Australia (email: \{xinyu.huang1, nan.yang\}@anu.edu.au).

Y. Fang is with the Department of Electrical and Electronic Engineering, University of Melbourne, Parkville, VIC 2010, Australia (e-mail: yuting.fang@unimelb.edu.au). stand on their own merits, the estimation schemes in MC have yet to be reviewed and summarized.

The estimation schemes investigated in current studies can be classified into two categories, namely, parameter estimation and channel estimation. Estimated parameters usually include the distance between the TX and the RX, the diffusion coefficient of molecules, the flow velocity in the MC environment, and so on. Among these parameters, distance estimation is the most popular research area in current studies. This is because the distance between nanomachines is one of the most pivotal parameters for the communication channel. Specifically, we summarize the significance of distance estimation in $\mathrm{MC}$ systems as follows:

- Distance estimation can be utilized to improve the channel performance since the distance affects the transmission rate. If a TX obtains the knowledge about the distance, it can adjust the number of released molecules to achieve a high probability of molecules arriving at a RX and avoid using too many molecules to reduce interferences, such as inter-symbol interference (ISI) and inter-link interference (ILI).

- In the application of targeted drug delivery [2], it is highly important to know the accurate location of the target site, e.g., a tumor, in the human body such that drugs are delivered to this site. The target site can be localized by estimating the relative distance between the tumor and nanomachines [6].

It is noted that in biological systems, there exist some techniques to determine the distance between two nanomachines in MC. For example, a cell can estimate the relative distance from an organism via producing a type of molecules to establish a chemical gradient [7].

Apart from distance estimation, estimating other parameters (i.e., the parameters excluding the distance) is also essential for some promising MC applications. For example, the MC system can be deployed in a blood-vessel environment for the healthcare application. In this application, estimating the blood flow velocity can help to measure the blood pressure. Also, estimating the diffusion coefficient of molecules can help to determine the blood composition and identify major changes in blood cell counts [8]. Furthermore, estimating the degradation rate of molecules can help to measure the $\mathrm{pH}$ level of blood since molecule degradation varies with the $\mathrm{pH}$ level [9, $\mathrm{Ch}$. 10]. Motivated by these benefits, some studies, e.g., [10]-[12], have proposed different methods for parameter estimation. Specifically, the estimated parameters include the number of emitted molecules, the degradation rate of molecules, the flow 
velocity, the diffusion coefficient of molecules, the release time of molecules, the clock offset between the TX and the RX, the start time of each symbol interval, and the signal-to-noise ratio (SNR).

Different from parameter estimation, channel estimation focuses on estimating the channel impulse response (CIR) in MC systems. Here, the CIR is defined as the probability of observing one molecule at the $\mathrm{RX}$ at time $t$ when molecules are impulsively released at time $t_{0}=0$. The CIR is important for the design of equalization and detection schemes in $\mathrm{MC}$ systems [13], [14]. Motivated by this importance, a few studies have investigated the estimation of the CIR via different methods, e.g., [15], [16].

In this paper, we divide estimation schemes into three categories: Distance estimation, estimation of other parameters (i.e., the parameters excluding the distance), and channel estimation. For each category, we provide detailed reviews of current studies. In particular, our major contributions are summarized as follows:

1) We examine the noise that may affect the performance of an estimation scheme and the metrics that are usually used to assess the performance of an estimation scheme.

2) We provide a detailed review on different distance estimation schemes. Then we compare the performance of different methods via numerical results by calculating the mean squared error (MSE).

3) We divide other parameters into environmental parameters, synchronization-related parameters, and SNR. Then we provide detailed reviews on each of them. Moreover, we review the pilot-based estimation scheme and the semi-blind CIR estimation scheme for channel estimation, and compare the performance of these two schemes.

4) We identify and discuss promising future research directions for parameter estimation and channel estimation.

The rest of this paper is organized as follows. In Section II we introduce a molecular communication via diffusion (MCvD) environment and present a summary of the noise that may impact on the estimation performance. In Section III, we present metrics for evaluating the performance of an estimation scheme. In Section IV we review the distance estimation schemes in current studies and compare the performance of different estimation schemes. In Section $\mathrm{V}$ we review the estimation of other parameters. In Section VI, we review channel estimation. In Section VII, we present future research directions for parameter estimation and channel estimation. Conclusions are drawn in Section VIII.

\section{Molecular Communication via Diffusion}

As most studies considered estimation schemes in an MCvD system 1 , we provide an explanation of this system as shown in Fig. 1, where one point TX communicates with one spherical RX with radius $r_{\mathrm{R}}$ in an unbounded three-dimensional (3D) environment. The RX center is at a distance $d$ away from the

\footnotetext{
${ }^{1}$ We also review studies that perform estimation in a non-MCvD environment in Section IV-B4
}

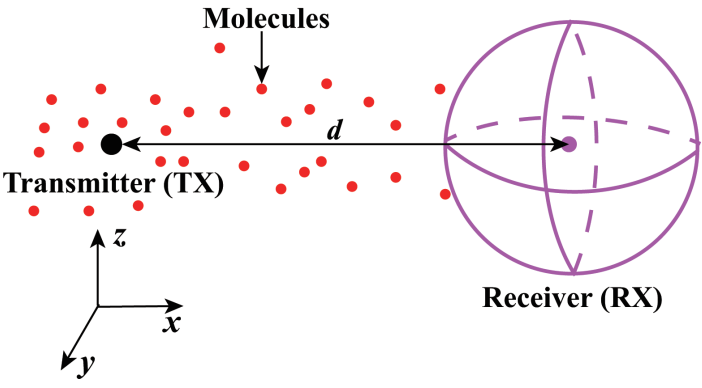

Fig. 1. Illustration of the MCvD environment where one point TX communicates with one spherical RX in a three-dimensional environment.

TX. We also consider the TX as a point source which releases molecules into the environment.

\section{A. Propagation Channel Modeling}

We assume that the propagation channel outside the TX and the RX is filled with a fluid medium. Once molecules are released, they diffuse randomly in the propagation environment. The movement speed of molecules is determined by the diffusion coefficient, denoted by $D$, that is affected by the temperature of the fluid medium, the dynamic viscosity of the fluid, and the Stoke's radius of molecules. In this paper, we assume that the fluid medium has uniform temperature and viscosity such that $D$ can be modeled as a constant value. It is noted that a more complex MC environment can incorporate flow with a constant velocity $v$ and degradation of molecules, i.e., molecules of type $A$ can degrade into some other molecular species $\hat{A}$ with a constant degradation rate $k$. $\hat{A}$ cannot be recognized by the RX.

\section{B. Receiver Modeling}

Current studies on estimation schemes focused on three types of RXs, i.e., transparent RX, fully-absorbing RX, and reactive RX. In this paper, we denote $h(t)$ as the CIR of the end-to-end channel.

1) Transparent $R X$ Modeling: The transparent $\mathrm{RX}$ does not impede the diffusion of molecules, nor interact with molecules. We count the number of free molecules that are within the RX volume as the received signal. The CIR of the transparent RX is given by [17, eq. (4)]

$$
h(t)=\frac{V_{\mathrm{Rx}}}{(4 \pi D t)^{\frac{3}{2}}} \exp \left(-\frac{d^{2}}{4 D t}\right),
$$

where $V_{\mathrm{RX}}$ is the volume of the $\mathrm{RX}$ and $V_{\mathrm{RX}}=\frac{4}{3} \pi r_{\mathrm{R}}^{3}$ for the spherical RX. It is noted that $h(t)$ incorporating the flow and degradation of molecules is given by [13, eq. (13)]. It is also noted that (1) is accurate when the RX is sufficiently far away from the TX, i.e., $d$ is very large relative to $r_{\mathrm{R}}$. Thus, it is reasonable to assume that the concentration of molecules at every point within the RX equals the concentration at the central point of the RX. If the RX is close to the TX, the 


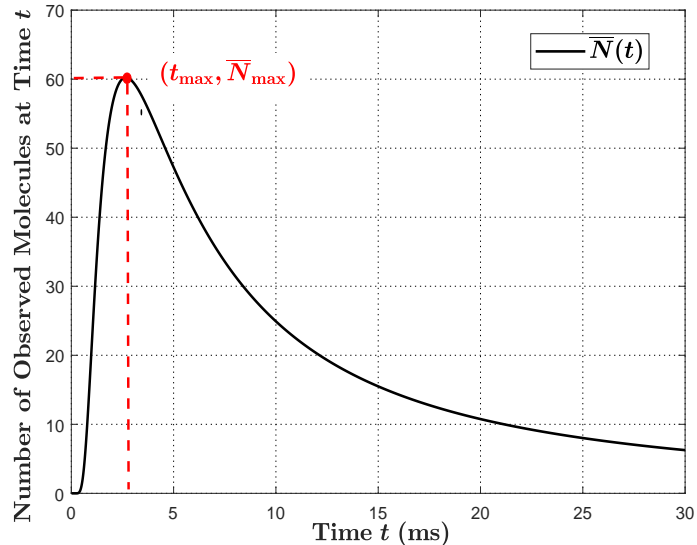

Fig. 2. The number of observed molecules within the transparent RX at time $t$ versus time $t$, where $N_{\mathrm{tx}}=10^{5}, r_{\mathrm{R}}=0.5 \mu \mathrm{m}, d=4 \mu \mathrm{m}$, and $D=1000 \mu \mathrm{m}^{2} / \mathrm{s}[19$.

uniform assumption of concentration does not hold. In this case, $h(t)$ is given by [18, eq. (27)]

$$
\begin{aligned}
h(t) & =\frac{1}{2}\left(\operatorname{erf}\left(\frac{r_{\mathrm{R}}-d}{\sqrt{4 D t}}\right)+\operatorname{erf}\left(\frac{r_{\mathrm{R}}+d}{\sqrt{4 D t}}\right)\right)+\frac{\sqrt{D t}}{r_{\mathrm{R}} \sqrt{\pi}} \\
& \times\left(\exp \left(-\frac{\left(r_{\mathrm{R}}-d\right)^{2}}{4 D t}\right)+\exp \left(-\frac{\left(r_{\mathrm{R}}+d\right)^{2}}{4 D t}\right)\right),
\end{aligned}
$$

where $\operatorname{erf}(\cdot)$ denotes the error function. We note that (1) is more widely applied in existing studies than (2), due to its simplicity, and (1) provides an accurate approximation for (2) if $r_{\mathrm{R}}<0.15 d$ [18].

We now denote $\bar{N}(t)$ as the expected number of molecules observed within the RX volume at time $t$. If an impulse of $N_{\text {tx }}$ molecules is released from the TX at time $t_{0}=0, \bar{N}(t)$ is given by $\bar{N}(t)=N_{\text {tx }} h(t)$. In Fig. 2, we plot $\bar{N}(t)$ versus time $t$ by adopting (1). From this figure, we observe a peak number of molecules observed within the RX. We denote the time for reaching the peak number of molecules observed as $t_{\max }$. By taking the derivative of (1) with respective to $t, t_{\max }$ is calculated as [19, eq. (6)]

$$
t_{\max }=\frac{d^{2}}{6 D} .
$$

By substituting (3) into (1), we obtain the peak CIR, denoted by $h_{\max }$, as

$$
h_{\max }=\left(d \sqrt{\frac{2 \pi e}{3}}\right)^{-3} V_{\mathrm{RX}} .
$$

We denote $\bar{N}_{\max }$ as the expected peak number of molecules observed within the RX. Then $\bar{N}_{\max }$ is given by $\bar{N}_{\max }=$ $N_{\text {tx }} h_{\text {max }}$.

2) Fully-Absorbing RX Modeling: In biological systems, many practical RX surfaces may interact with the molecules of interest, e.g., by providing binding sites for absorption or other reactions [20]. Hence, the transparent RX model is oversimplified. One practical RX model is the fully-absorbing RX. In this model, the RX absorbs molecules as soon as they hit the surface. The fully-absorbing RX counts the total number of molecules absorbed as the received signal, where the CIR is given by [21, eq. (23)]

$$
h(t)=\frac{r_{\mathrm{R}}}{d} \operatorname{erfc}\left(\frac{d-r_{\mathrm{R}}}{\sqrt{4 D t}}\right) .
$$

3) Reactive RX Modeling: Different from the fullyabsorbing RX, molecules that reach the RX may participate in a reversible bimolecular second-order reaction with receptors over the RX membrane. This type of RX is named as the reactive $\mathrm{RX}$ whose received signal is the number of activated receptors. The CIR of the reactive RX is given by [22, eq. (29)] and is omitted here due to its complex format.

\section{Noise Modeling}

We next review some factors that affect the performance of estimation and treat these factors as noise during the estimation process.

1) Statistical Distribution of Received Signal: Due to the random diffusion (RD) of molecules, the number of molecules observed at the RX is a random variable (RV) [23]. This randomness influences the performance of estimation. Due to the independent diffusion of molecules, any given molecule released by the TX is observed by the RX with a probability of $h(t)$. A binary state model applies and the number of molecules observed at time $t$, denoted by $N_{\mathrm{ob}}(t)$, follows a binomial distribution with $N_{\text {tx }}$ trails and success probability $h(t)$. This is mathematically expressed as

$$
N_{\mathrm{ob}}(t) \sim \mathcal{B}\left(N_{\mathrm{tx}}, h(t)\right),
$$

where $\mathcal{B}(N, p)$ represents a binomial distribution. Unfortunately, the binomial distribution is cumbersome to work with in MC systems. Therefore, current studies usually approximate binomial distribution as two distributions for the sake of mathematical tractability, described as follows:

(a) Poisson distribution: When the number of trials $N_{\mathrm{tx}}$ is large and the success probability $h(t)$ is small, $N_{\mathrm{ob}}(t)$ can be approximated as a Poisson RV, given by

$$
N_{\mathrm{ob}}(t) \sim \mathcal{P}\left(N_{\mathrm{tx}} h(t)\right),
$$

where $\mathcal{P}(\varphi)$ represents the Poisson distribution with the mean of $\varphi$. Based on (7), the probability mass function (PMF) of the Poisson RV $N_{\mathrm{ob}}(t)$ is written as

$$
\operatorname{Pr}\left(N_{\mathrm{ob}}(t)=\xi\right)=\frac{\left(N_{\mathrm{tx}} h(t)\right)^{\xi} \exp \left(-N_{\mathrm{tx}} h(t)\right)}{\xi !},
$$

where $\operatorname{Pr}(\cdot)$ stands for the probability.

(b) Gaussian distribution: If the expected number of molecules observed, i.e., $\bar{N}(t)$, is sufficiently large, we can apply the central limit theorem and approximate $N_{\text {ob }}(t)$ as a Gaussian RV, given by

$$
N_{\mathrm{ob}}(t) \sim \mathcal{N}\left(N_{\mathrm{tx}} h(t), N_{\mathrm{tx}} h(t)(1-h(t))\right) .
$$

The probability density function (PDF) of $N_{\mathrm{ob}}(t)$ is given by

$$
\operatorname{Pr}\left(N_{\mathrm{ob}}(t)=\xi\right)=\frac{\exp \left(-\frac{\left(\xi-N_{\mathrm{tx}} h(t)\right)^{2}}{2 N_{\mathrm{tx}} h(t)(1-h(t))}\right)}{\sqrt{2 \pi N_{\mathrm{tx}} h(t)(1-h(t))}} .
$$


2) External Additive Noise: In MC systems, the intended TX may not be the only source of molecules. Other sources, referred to as external sources, may also release the same type of molecules that influence the observation at the RX and affects the estimation performance. We detail some examples of external sources as follows:

(a) Multiuser interference: Noisy molecules are emitted by TXs in other MC systems.

(b) Unintended leakage: Molecules can be leaked from membrane-bound containers, e.g., vesicles, within transceivers. A rupture can result in a steady or sudden release of molecules [24].

(c) Output from unrelated biochemical processes: Biocompatibility of the MC system may require the selection of naturally-occurring molecules. Therefore, other processes that produce the same type of molecules are noisy sources for the considered MC system. For example, calcium is commonly used as a messenger molecule within cellular systems [25, Ch. 16]. If calcium is applied as signaling molecules of the MC system in the biological environment, the naturally-occurring calcium would impact the $\mathrm{MC}$ system.

(d) Unintended reception of other molecules: Molecules that are highly similar to intended molecules may be recognized by the RX. For example, the receptors at the RX may bind to other molecules that have a very similar shape and size to intended molecules [25, Ch. 4].

We denote $N_{\text {sig }}(t)$ as the intended observed molecules and $n(t)$ as the observed noise molecules. Since intended molecules and noise molecules are indistinguishable, the total number of molecules observed at the $\mathrm{RX}$ at time $t$ is given by

$$
N_{\mathrm{ob}}(t)=N_{\mathrm{sig}}(t)+n(t) .
$$

The analysis of the statistical distribution of $n(t)$ is built upon following assumptions:

A1) We denote $\bar{n}$ as the expected number of noise molecules observed within the RX. We assume that $\bar{n}$ is constant over the entire observation time.

A2) The observation of one noise molecule at the RX is independent of observations of other noise molecules.

A3) The uniform concentration assumption holds for noise molecules at the RX.

Based on A1)-A3), we model the number of observed noise molecules as a Poisson RV, due to the law of rare events (LRE) [26], i.e., $n(t) \sim \mathcal{P}(\bar{n})$.

3) ISI \& ILI: ISI exists when the TX transmits multiple symbols to the RX. Due to the RD of molecules, the molecules from previously sent symbols may arrive at the RX in the current symbol interval, which influences the estimation in the current symbol interval. ILI exists for the multiple-input multiple-output (MIMO) MC system, where one TX - RX channel is influenced by molecules released from other channels.

\section{Performance Metrics for Estimation Schemes}

In this section, we review some metrics that are usually applied to evaluate the performance of an estimation scheme.

\section{A. $M S E$}

The MSE is usually applied to assess the quality of an estimation scheme. We denote $\theta$ as the unknown parameter and $\hat{\theta}$ as the estimated value of $\theta$. The MSE of an estimation scheme is defined as [27]

$$
\operatorname{MSE}(\hat{\theta})=\mathbb{E}\left[(\hat{\theta}-\theta)^{2}\right],
$$

where $\mathbb{E}[\cdot]$ represents the expectation. The MSE can be written as the sum of the variance and squared bias of the estimation scheme, which is [28]

$$
\operatorname{MSE}(\hat{\theta})=\operatorname{Var}(\hat{\theta})+\operatorname{Bias}(\hat{\theta}, \theta)^{2},
$$

where the variance is $\operatorname{Var}(\hat{\theta})=\mathbb{E}\left[(\hat{\theta}-\mathbb{E}[\hat{\theta}])^{2}\right]$ and the squared bias is $\operatorname{Bias}(\hat{\theta}, \theta)^{2}=(\mathbb{E}[\hat{\theta}]-\theta)^{2}$. For any unbiased estimation scheme, $\mathbb{E}[\hat{\theta}]=\theta$. Thus, the MSE equals the variance.

\section{B. Cramer-Rao Lower Bound (CRLB)}

The CRLB is a lower bound on the variance of any unbiased estimation scheme [29, Ch. 3]. An estimation scheme is called the minimum-variance unbiased (MVU) estimator if its MSE attains the CRLB. Therefore, the CRLB provides insights into the comparison of estimation schemes and the prediction of the performance of the MVU estimator.

We consider an $M$-point data set $\mathbf{x}=\left[x_{1}, x_{2}, \cdots, x_{M}\right]$ that depends on an unknown parameter vector $\boldsymbol{\theta}$, where $\boldsymbol{\theta}$ contains $L$ unknown parameters as $\boldsymbol{\theta}=\left[\theta_{1}, \theta_{2}, \cdots, \theta_{L}\right]$. Hence, the date set $\mathbf{x}$ is applied to determine $\boldsymbol{\theta}$. To mathematically model the data set, we define a PDF as $p(\mathbf{x} ; \boldsymbol{\theta})$ that is parameterized by the unknown parameter vector $\boldsymbol{\theta}$. For the CRLB to exist, the regularity condition must be satisfied, which is given by [29, Ch. 3]

$$
\mathbb{E}\left[\frac{\partial \ln p(\mathbf{x} ; \boldsymbol{\theta})}{\partial \boldsymbol{\theta}}\right]=\mathbf{0}, \text { for all } \boldsymbol{\theta},
$$

where the expectation is taken with respect to $p(\mathbf{x} ; \boldsymbol{\theta})$. We denote $\hat{\boldsymbol{\theta}}$ as the estimated parameter vector of $\boldsymbol{\theta}$. The covariance matrix of any unbiased estimation scheme $\hat{\boldsymbol{\theta}}$, denoted by $\mathbf{C}_{\hat{\boldsymbol{\theta}}}$, satisfies [29, eq. (3.24)]

$$
\mathbf{C}_{\hat{\boldsymbol{\theta}}}-\mathbf{I}^{-1}(\boldsymbol{\theta}) \geq \mathbf{0},
$$

where $\mathbf{I}(\boldsymbol{\theta})$ is an $L \times L$ Fisher information matrix, given by

$$
[\mathbf{I}(\boldsymbol{\theta})]_{i j}=-\mathbb{E}\left[\frac{\partial^{2} \ln p(\mathbf{x} ; \boldsymbol{\theta})}{\partial \theta_{i} \partial \theta_{j}}\right],
$$

with $i=1,2, \cdots, L$ and $j=1,2, \cdots, L$. The derivatives are evaluated at the true value of $\boldsymbol{\theta}$. The CRLB on $\theta_{l}$ is found as the $[l, l]$ element of the inverse of $\mathbf{I}(\boldsymbol{\theta})$, which is

$$
\operatorname{Var}\left(\hat{\theta}_{l}\right) \geq\left[\mathbf{I}^{-1}(\boldsymbol{\theta})\right]_{l l} .
$$

When only a single papermeter $\theta$ is unknown, (14) is simplified as

$$
\mathbb{E}\left[\frac{\partial \ln p(\mathbf{x} ; \theta)}{\partial \theta}\right]=0, \text { for all } \theta,
$$


where $p(\mathbf{x} ; \theta)$ is the PDF parameterized by the unknown parameter $\theta$. We simplify (16) as [29, eq. (3.6)]

$$
I(\theta)=-\mathbb{E}\left[\frac{\partial^{2} \ln p(\mathbf{x} ; \theta)}{\partial \theta^{2}}\right],
$$

where $I(\theta)$ is the Fisher information. The CRLB for the unbiased estimation of $\theta$ is $\operatorname{Var}(\hat{\theta}) \geq I^{-1}(\theta)$.

\section{Hammersley-Chapman-Robbins Lower Bound (HCRLB)}

The HCRLB is a lower bound on the variance of any unbiased estimation scheme for a function parameterized by the unknown parameter [30]. Compared to the CRLB, the HCRLB is tighter and does not need to satisfy the regularity condition, while the computation is more complex. We denote $g(\theta)$ as a function of the unknown parameter $\theta$ and $\hat{g}(\theta)$ as an estimated function of $g(\theta)$. The HCRLB on the variance of $\hat{g}(\theta)$ is given by

$$
\operatorname{Var}(\hat{g}(\theta)) \geq \sup _{\Delta} \frac{[g(\theta+\Delta)-g(\theta)]^{2}}{\mathbb{E}\left[\frac{p(\mathbf{x} ; \theta+\Delta)}{p(\mathbf{x} ; \theta)}-1\right]^{2}},
$$

where sup stands for supremum. If we set $g(\theta)=\theta$ and substitute it into 20, we obtain the HCRLB on $\operatorname{Var}(\hat{\theta})$. In (20), the HCRLB converges to the CRLB when $\Delta \rightarrow 0$. The HCRLB can be applied to a wider range of problems. For example, if $p(\mathbf{x} ; \theta)$ is non-differentiable, the Fisher information is not defined. Hence, the CRLB does not exist. However, the HCRLB may exist under this condition.

\section{Distance Estimation}

In this section, we review the current studies on the estimation of the distance between the TX and the RX in MC systems. We classify the current studies about distance estimation into two-way estimation and one-way estimation in Section IV-A and Section IV-B, respectively. We summarize different distance estimation schemes in Table It is noted that some studies focused on the one-dimensional (1D) environment while other studies focused on the 3D environment. To facilitate the comparison, we consider all estimation schemes in the 3D environment via following the estimation process and replacing the CIR in 1D with $h(t)$ given in (1), (2), or (5) for different types of RXs.

\section{A. Two-Way Estimation}

Two-way estimation schemes were proposed in [10] to estimate the distance between two transceivers that are labeled as $T$ and $R$, respectively. Specifically, $T$ first releases an impulse of molecules of type $A$ at time $t_{0}$. The diffusion coefficient of molecules $A$ is $D_{A}$. When $R$ detects the molecules $A$ at time $t_{1}$, it immediately transmits a feedback signal of an impulse of molecules $B$ whose diffusion coefficient is $D_{B}$. At time $t_{2}, T$ detects the molecules $B . T$ and $R$ are regarded as transparent RXs when they detect molecules $B$ and $A$, respectively.
1) Round Trip Time (RTT) Protocols: In RTT protocols, $T$ measures the RTT that is the sum of time required for the transmission from $T$ to $R$ and for the transmission from $R$ to $T$. The first estimation scheme is named as the RTT protocol from peak concentration (RTT-P). In RTT-P, $T$ transmits at time $t_{0}$ and $R$ detects the peak concentration of molecules $A$ from $T$ at time $t_{1}$. According to (3), we can obtain the relationship between $t_{1}-t_{0}$ and $d$. $T$ detects the peak concentration of the feedback signal with type $B$ molecules at time $t_{2}$. Similarly, we can obtain the relation between $t_{2}-t_{1}$ and $d$ based on (3). Accordingly, the distance $d$ is estimated as a function of the RTT $t_{2}-t_{0}$ as

$$
\hat{d}=\sqrt{\frac{6 D_{A} D_{B}}{D_{A}+D_{B}}\left(t_{2}-t_{0}\right)},
$$

where $\hat{d}$ is the estimated value of $d$.

The second estimation scheme was named as the RTT protocol from threshold concentration (RTT-T). Different from RTT-P, RTT-T defines threshold concentrations $H_{A}$ and $H_{B}$ for $R$ and $T$ to detect the number of molecules observed, respectively. It is assumed that $T$ transmits $N_{\mathrm{tx}}^{A}$ number of molecules $A$ and $R$ transmits $N_{\mathrm{tx}}^{B}$ number of molecules $B . R$ records $t_{1}$ when the number of molecules observed reach the threshold concentration $H_{A}$, i.e., $\left.\bar{N}\left(t_{1}-t_{0}\right)\right|_{D=D_{A}, N_{\mathrm{tx}}=N_{\mathrm{tx}}^{A}}=$ $H_{A}$ by assuming $\bar{N}(t)=N_{\mathrm{ob}}(t)$. Similarly, $T$ records $t_{2}$ when the number of molecules observed reach the threshold concentration $H_{B}$, i.e., $\left.\bar{N}\left(t_{2}-t_{1}\right)\right|_{D=D_{B}, N_{\mathrm{tx}}=N_{\mathrm{txx}}^{B}}=H_{B}$. If $D_{A}=D_{B}, N_{\mathrm{tx}}^{A}=N_{\mathrm{tx}}^{B}$, and $H_{A}=H_{B}, d$ is estimated as a function of the RTT $t_{2}-t_{0}$ as

$$
\hat{d}=\sqrt{D_{A}\left(t_{2}-t_{0}\right) \ln \left(\frac{V_{\mathrm{RX}}^{2}\left(N_{\mathrm{tx}}^{A}\right)^{2}}{8 \pi^{3} D_{A}^{3} H_{A}^{2}\left(t_{2}-t_{0}\right)^{3}}\right)} .
$$

2) Signal Attenuation Protocol from Peak Concentration (SA-P): In SA-P, $T$ transmits type $A$ molecules to $R$, and $R$ measures the peak concentration, denoted by $N_{\mathrm{ob}, \mathrm{m}}^{A}$. According to (4), we can obtain the relationship between $N_{\mathrm{ob}, \mathrm{m}}^{A}$ and $d$ by replacing $\bar{N}_{\text {max }}$ with $N_{\mathrm{ob}, \mathrm{m}}^{A}$. Similarly, $R$ transmits type $B$ molecules and $T$ measures the peak concentration, denoted by $N_{\mathrm{ob}, \mathrm{m}}^{B}$. By assuming $N_{\mathrm{tx}}^{B}=N_{\mathrm{ob}, \mathrm{m}}^{A}, \hat{d}$ is obtained as

$$
\hat{d}=\sqrt{\frac{3}{2 \pi e}}\left(\frac{N_{\mathrm{tx}}^{A} V_{\mathrm{RX}}^{2}}{N_{\mathrm{ob}, \mathrm{m}}^{B}}\right)^{\frac{1}{6}} .
$$

The estimation performance of SA has been shown in [19].

3) Merits and Drawbacks: In this subsection, we summarize the merits and drawbacks of the two-way estimation. One merit of this estimation is that the synchronization between two transceivers is not required. For both RTT and SA protocols, only the time period of two-way transmission, the number of emitted molecules, and peak observed concentration at $T$ are required. Despite this merit, there are several drawbacks. The first drawback is that this estimation is time-consuming since it requires two-way transmission. The second one is that this estimation requires $R$ to immediately send the feedback signal when it detects the type of molecules $A$, which is challenge for a nanomachine. The third drawback is that the instantaneous observation at the RX is used to approximate its expectation, which affects the estimation performance. 
TABLE I

Distance Estimation SCHEMES FOR MC Systems

\begin{tabular}{|c|c|c|c|c|c|c|c|c|}
\hline Name & References & Performance & $\begin{array}{c}\text { Computational } \\
\text { Complexity }\end{array}$ & Synchronization & Noise & $\begin{array}{c}\text { TX } \\
\text { Waveform }\end{array}$ & RX Type & Environment \\
\hline RTT & 【10,, 31$]$ & Moderate & Low & Not Required & RD & Impulse & Transparent & 1D \\
\hline SA & -10] & Low & Low & Not Required & $\mathrm{RD}$ & Impulse & Transparent & $1 \mathrm{D}$ \\
\hline \multirow{3}{*}{$\begin{array}{l}\text { Peak-Based } \\
\text { (One Type of } \\
\text { Molecule) }\end{array}$} & 32,33 & Moderate & Low & Required & $\mathrm{RD}$ & Impulse & Transparent & $1 \mathrm{D}, 2 \mathrm{D}$ \\
\hline & {$[11]$} & Moderate & Low & Required & $\mathrm{RD}$ & Rectangular & Transparent & $3 \mathrm{D}$ \\
\hline & 34 & Moderate & Low & Required & $\mathrm{RD}$ & Impulse & $\begin{array}{l}\text { Transparent, } \\
\text { Ring-Shaped }\end{array}$ & $\begin{array}{c}\text { Cylindrical, } \\
\text { Poiseuille Flow }\end{array}$ \\
\hline $\begin{array}{c}\text { Peak-Based } \\
\text { (Two Types of } \\
\text { Molecules) }\end{array}$ & 35, 36 & Low & Low & Not Required & $\mathrm{RD}$ & Impulse & Transparent & 1D \\
\hline \multirow{3}{*}{ ML } & 19, 37 & High & High & Required & $\mathrm{RD}$ & Impulse & Transparent & $\begin{array}{c}\text { 3D, Flow, } \\
\text { Molecules } \\
\text { Degradation }\end{array}$ \\
\hline & 38 & High & High & Required & ISI & Impulse & Transparent & $3 \mathrm{D}$ \\
\hline & 39 & High & High & Required & $\mathrm{RD}$ & $\begin{array}{l}\text { Impulse, } \\
\text { Diffusive }\end{array}$ & $\begin{array}{c}\text { Transparent, } \\
\text { Diffusive } \\
\end{array}$ & 3D \\
\hline $\begin{array}{c}\text { Fraction of } \\
\text { Absorbed Molecules }\end{array}$ & 40] & N/A & High & $\begin{array}{c}\text { Not Required } \\
\& \text { Required }\end{array}$ & ISI, RD & Impulse & Fully-Absorbing & 3D \\
\hline Data Fitting & 41 & N/A & High & Required & $\mathrm{RD}$ & Impulse & $\begin{array}{c}\text { Multiple, } \\
\text { Fully-Absorbing }\end{array}$ & $3 \mathrm{D}$ \\
\hline Macro-scale & [42] & N/A & High & Required & N/A & Sprayer & MQ-3 Sensor & Tabletop \\
\hline
\end{tabular}

\section{B. One-Way Estimation}

Due to the aforementioned drawbacks of the two-way estimation, most current studies have focused on the acquisition of the distance information from the received signal only, referred to as one-way estimation.

1) Peak-Based Estimation: In this subsection, we review the studies that perform the estimation via measuring the peak received signal or the time for reaching the peak received signal.

First, [32] performed the estimation based on the peak number of observed molecules, denoted by $N_{\mathrm{ob}, \mathrm{m}}$, at the transparent RX. According to (4), the distance $d$ is estimated as

$$
\hat{d}=\sqrt{\frac{3}{2 \pi e}}\left(\frac{V_{\mathrm{RX}} N_{\mathrm{tx}}}{N_{\mathrm{ob}, \mathrm{m}}}\right)^{\frac{1}{3}} .
$$

The estimation performance of this method is affected by the $\mathrm{RD}$ of molecules, since the instantaneous observation is used to approximate its expectation.

Second, [11] considered, for the first time, the release of molecules from the TX as a rectangular pulse, where the CIR at the transparent RX is denoted by $h_{\mathrm{rec}}(t)$. By taking the derivative of $h_{\text {rec }}(t)$ with respect to $t, d$ can be estimated via measuring the time for reaching the peak concentration at the RX as

$$
\hat{d}=\sqrt{\frac{6 D t_{\max }\left(t_{\max }-T_{\mathrm{e}}\right)}{T_{\mathrm{e}}} \ln \left(\frac{t_{\max }}{t_{\max }-T_{\mathrm{e}}}\right)},
$$

where $T_{\mathrm{e}}$ is the emission duration.

As $t_{\max }$ is involved in the estimation of [11], a perfect synchronization between the TX and the RX is required. In [35], [36], the authors proposed a low-complexity scheme that does not require the synchronization via adopting two types of molecules. In this scheme, the TX releases type $A$ molecules at time $t_{0}$ and the RX records the time of peak concentration of molecules $A$, denoted by $t_{\max }^{A}$. Similarly, the TX transmits type $B$ molecules at $t_{1}$, and the RX records the time of peak

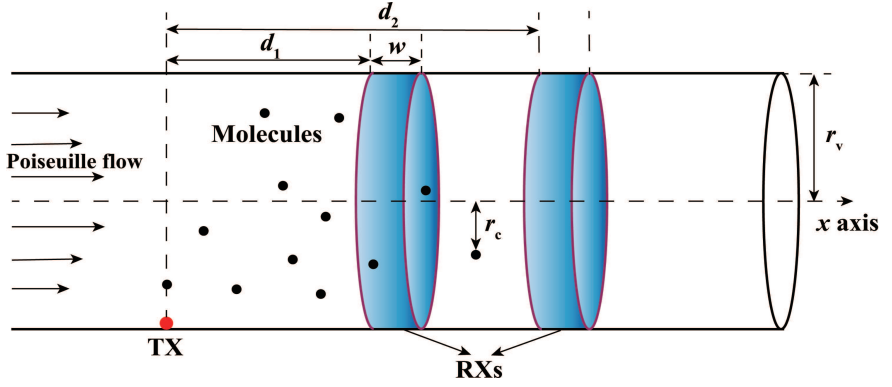

Fig. 3. Estimation in a cylindrical diffusive $\mathrm{MC}$ environment by using ringshaped RXs; reproduced based on [34].

concentration of molecules $B$, denoted by $t_{\max }^{B}$. According to (3), the relation between $t_{\max }^{A}$ and $d$, and $t_{\max }^{B}$ and $d$ are obtained. Thus, $d$ is estimated as

$$
\hat{d}=\sqrt{\frac{6 D_{A} D_{B}\left(\Delta t_{\mathrm{Rx}}-\Delta t_{\mathrm{Tx}}\right)}{D_{A}-D_{B}}},
$$

where $\Delta t_{\mathrm{Rx}}=t_{\max }^{B}-t_{\max }^{A}$ and $\Delta t_{\mathrm{Tx}}=t_{1}-t_{0}$. We note that $\Delta t_{\mathrm{RX}}$ and $\Delta t_{\mathrm{TX}}$ are based on the time measurements at the RX and the TX, respectively. Therefore, synchronization is not required.

In [10], [11], [32], [35], [36], estimation is performed in an unbounded environment. Different from these studies, [34] considered a cylindrical MC environment whose surface is reflecting as shown in Fig. 3. Within the cylindrical environment, a point TX releases molecules and two ring-shaped RXs with a certain width $w$ are located on the cylinder perimeter with a radius $r_{\mathrm{v}}$ that is the same as the cylinder's radius. After the molecules are released, they diffuse randomly with a constant diffusion coefficient $D$ and subject to the Poiseuille flow [43]. As the cylindrical MC environment is a good approximation of the blood vessel environment, it has been widely investigated in existing studies, e.g., [34], [44], [45]. Due to the existence of the Poiseuille flow, advection and diffusion can both transport molecules. In [34], Turan et al. considered a diffusion-domain movement and only focused on the movement in the $x$ axis, 
i.e., the channel is approximated as a 1D environment. The authors performed the estimation in two scenarios. The first scenario is that the emission time of molecules is known. By measuring the time of peak concentration, the distance can be estimated by a single RX as

$\hat{d}_{1} \approx \frac{-w+\sqrt{w^{2}-8\left(w v_{m} t_{\max }-2\left(v_{m} t_{\max }\right)^{2}-4 D_{\mathrm{e}} t_{\max }\right)}}{4}$,

where $D_{\mathrm{e}}$ is the effective diffusion coefficient and $v_{m}$ is the average flow velocity. The second scenario is that the emission time is unknown. Thus, $t_{\max }$ is unknown and 27) contains two unknown parameters, i.e., $d_{1}$ and $t_{\max }$. In this scenario, $d_{1}$ is estimated by using two RXs. Similar to 27), an equation containing $d_{1}$ and $t_{\max }$ can be obtained at the second RX. Thus, $d_{1}$ can be mathematically derived by jointly solving these two equations.

2) Maximum Likelihood (ML) Estimation: The ML estimation is to find $\hat{\theta}$ that maximizes the joint observation likelihood, which usually achieves high accuracy, but requires high computational complexity and the perfect synchronization between the TX and the RX. The authors in [19], [37] considered $x_{m}$, i.e., the $m$ th data of the data set $\mathbf{x}$, as the number of molecules observed at the transparent RX at time $t_{m}$ when molecules are released at time $t_{0}$. It is assumed that each observation is independent and follows a Poisson distribution. Thus, $p(\mathbf{x} ; \theta)$ is given by

$$
p(\mathbf{x} ; \theta)=\prod_{m=1}^{M} \frac{\left(N_{\mathrm{tx}} h\left(t_{m}\right)\right)^{x_{m}}}{x_{m} !} \exp \left(-N_{\mathrm{tx}} h\left(t_{m}\right)\right) .
$$

Using (28), $d$ is estimated by taking the partial derivative of $p(\mathbf{x} ; \theta)$ with respect to $d$ and setting it equal to 0 . Moreover, [19] derived the CRLB on the variance of $\hat{d}$.

It is noted that [19], [37] only considered the transmission of one symbol from the TX to the RX. Different from that, [38] considered multiple symbols transmitted from the TX to the transparent RX, where ISI exists and may impact the estimation performance. Assuming that the RX makes one observation in each symbol interval, $p(\mathbf{x} ; \theta)$ is the joint PDF of multiple observations. Based on $p(\mathbf{x} ; \theta), d$ is estimated.

Previous studies have focused on the distance estimation in a static MC system. Instead of that, [39] considered estimating the initial distance, i.e., the distance between the TX and the $\mathrm{RX}$ at the initial moment, in a diffusive mobile MC scenario, where both TX and RX diffuse with constant diffusion coefficients $D_{\mathrm{TX}}$ and $D_{\mathrm{RX}}$, respectively. A novel two-step scheme was proposed to estimate the initial distance, denoted by $d_{0}$. We denote $d(t)$ as the distance between the TX and the RX at time $t$, and the PDF of $d(t)$ is given by [39, eq. (13)], where $d_{0}$ is a parameter of the PDF of $d(t)$. Moreover, [39] proved that the ML estimation based on the joint PDF of multiple values of $d(t)$ can be simplified as performing the estimation based on the PDF of the first value of $d(t)$, i.e., $d\left(t_{1}\right)$. Therefore, the first step is to estimate the stochastic distance $d\left(t_{1}\right)$, where the estimation scheme is similar to [19]. The second step is to estimate $d_{0}$ by finding $\hat{d}_{0}$ that maximizes the PDF of $d\left(t_{1}\right)$.

As the closed-form expression for $\hat{d}$ is difficult to derive in the ML estimation, [38] and [39] used the Newton-Raphson

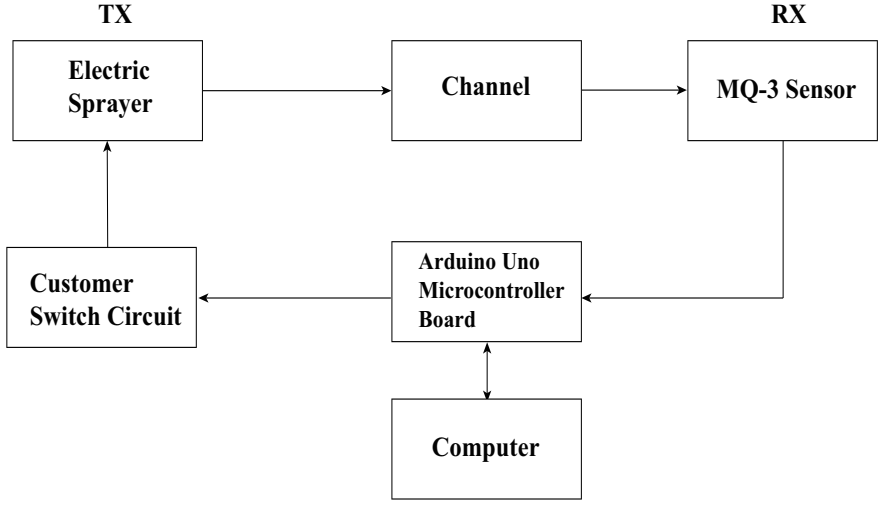

Fig. 4. Block diagram of the experimental setup in [42].

method, which is a method to find successively better approximations to the roots of a real-valued function, in order to calculate $\hat{d}$.

3) Non-transparent $R X s$ : In this section, we review estimation schemes in an environment with non-transparent RXs. Wang et al. in [40] proposed an estimation scheme by applying a fully-absorbing RX. The RX performs estimation by counting the number of molecules absorbed within a time interval. Specifically, an algorithm was proposed when the TX and the RX are unsynchronized. Moreover, the authors considered two optimization methods to improve the performance of estimation. The first method is using molecules with a large diffusion coefficient and the second method is increasing the number of emitted molecules.

Different from [40], the authors in [41] performed the estimation in an environment with multiple fully-absorbing RXs. As one fully-absorbing RX would impact molecules absorbed by other fully-absorbing RXs, an accurate derivation for the number of molecules absorbed at each RX is cumbersome. Miao et al. in [41] adopted a curve fitting method to obtain the expression for the number of absorbed molecules. Specifically, [41] used the nonlinear least squares method for curve fitting to obtain the distance, where the Levenberg-Marquardt (LM) method [46] is adopted.

4) Macro-scale MC Systems: Previous studies focused on the distance estimation in micro-scale ( $\mathrm{nm}$ to $\mu \mathrm{m}$ ) MC systems. With this focus, these studies have considered an ideal channel model where the transmitted molecules do not have an initial velocity, the molecules move according to Brownian motion, and the TX and the RX perfectly transmit and receive signals. In nature, $\mathrm{MC}$ also exists in the macro-scale $(\mathrm{cm}$ to $\mathrm{m}$ ) environment. For example, animals like bees, flies and ants use pheromone to send messengers over several meters. Against this background, [42] investigated, for the first time, the distance estimation in the macro-scale environment. Instead of analyzing a theoretical model, [42] established an experimental setup similar to the tabletop platform in [47]. Fig. 4 shows the block diagram of the experimental setup. The TX is an electric sprayer controlled by a micro-controller via a custom switch circuit to release ethyl alcohol molecules. The RX receives the molecular signal with an MQ-3 alcohol sensor. The TX and the RX are both controlled by an Arduino Uno micro-controller board which is connected to a computer. 


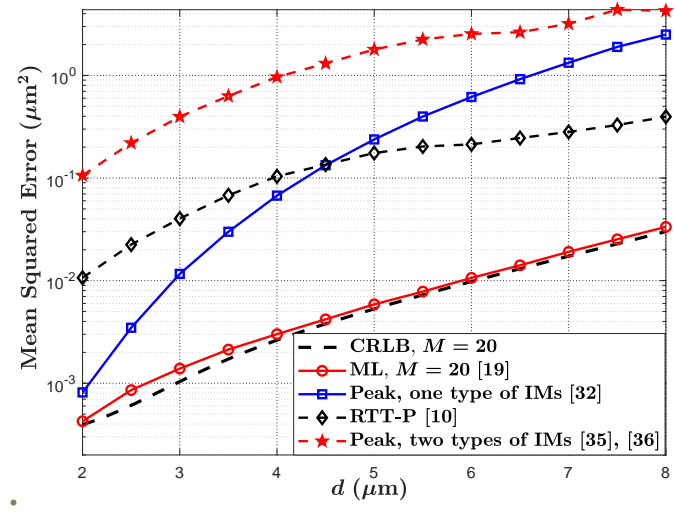

Fig. 5. The MSE of different estimation schemes versus $d$.

Five different practical methods were proposed for distance estimation at the RX. The first two methods adopt the supervised machine learning, where multivariate linear regression and neural network regression are used. These methods use the extracted features, e.g., rise time on the rising edge of the measured signal, from the received molecular signals as inputs. The other three methods analyze the collected data, which are less complex but less accurate than machine learning methods. The first data analysis method employs the received power to estimate the distance. The second data analysis method employs the peak time of the received signal. The third method combines the power and peak time of the received signal to estimate the distance.

\section{Performance Comparison}

In this subsection, we present numerical results to compare the distance estimation performance of different estimation schemes via calculating their MSE. Particle-based simulation is used to simulate the random propagation of molecules [48]. The simulation time step is $\Delta t_{\text {sim }}=0.0001 \mathrm{~s}$ and all results are averaged over 10,000 realizations. Throughout this subsection, we set $N_{\mathrm{tx}}=N_{\mathrm{tx}}^{A}=N_{\mathrm{tx}}^{B}=10^{5}, r_{\mathrm{R}}=0.5 \mu \mathrm{m}$, $D=D_{A}=1000 \mu \mathrm{m}^{2} / \mathrm{s}$, and $D_{B}=500 \mu \mathrm{m}^{2} / \mathrm{s}$ [12].

In Fig. 5, we plot the MSE of different estimation schemes versus the distance $d$. Here, estimation schemes include the RTT-P [10], the ML estimation [19], the peak-based estimation applying a single type of molecules [32] and two types of molecules [35], [36]. We also plot the CRLB as a lower bound to assess the performance of each estimation scheme. For the CRLB and ML estimation, we apply $M=20$ observations. First, we observe that the MSE of ML estimation almost attains the CRLB and hence achieves the best performance. Second, we observe that the peak-based estimation using one type of molecules and RTT-P achieves a moderate performance. The peak-based estimation using one type of molecules outperforms the RTT-P when $d$ is small. When $d$ is large, the advantage of RTT-P becomes more obvious. Third, the peakbased estimation using two types of molecules achieves the worst performance since it calculates the time difference, but does not need synchronization.

\section{Estimation of Other Parameters}

In this section, we review the current studies on the estimation of other parameters (i.e. the parameters excluding distance). We classify these parameters into three categories. The first category is referred to as the environmental parameters that are related to channel and TX properties, e.g., the diffusion coefficient and the number of released molecules. The second category is referred to as the synchronizationrelated parameters that are estimated to achieve synchronization between the TX and the RX. The third category is referred to as the SNR. We summarize the studies that estimate these parameters in Table $\Pi$

\section{A. Environmental Parameters}

In this subsection, we focus on the studies that estimate environmental parameters. Specifically, environmental parameters include the distance between the TX and the RX, release time of molecules, diffusion coefficient of molecules, degradation rate of molecules, flow velocity, and the number of released molecules.

In [12], Noel et al. considered a joint environmental parameter estimation, where the unknown parameter vector $\boldsymbol{\theta}$ contains a single or multiple parameters. By assuming each observation of the received signal at the transparent RX is independent and follows a Poisson distribution, [12] derived the Fisher information matrix $\mathbf{I}(\boldsymbol{\theta})$ and the CRLB when a single unknown parameter exists or multiple unknown parameters exist. Moreover, the ML estimation was applied to estimate the unknown parameters.

In [49], the authors evaluated the HCRLB for a special case via setting $g(\theta)=N_{\mathrm{tx}}$, i.e., the HCRLB on the variance of the estimated number of released molecules. Similar to (28), [49] derived $p(\mathbf{x} ; \theta)$ as the joint PMF of the received signals at the transparent RX.

The authors in [12], [49] regarded $N_{\mathrm{tx}}$ as a constant value in the estimation scheme. Different from that, [52] regarded $N_{\mathrm{tx}}$ as a RV with the mean of $\mu_{\mathrm{tx}}$ and variance of $\sigma_{\mathrm{tx}}^{2}$. The authors assumed that $\mu_{\mathrm{tx}}$ is pre-determined and estimated $\sigma_{\mathrm{tx}}^{2}$ by emitting multiple impulses of molecules. The RX detects the received signal at the peak time. By considering that $V_{\mathrm{RX}} \rightarrow \infty$, i.e., the molecular concentration is perfectly sensed over the entire environment, $\hat{\sigma}_{\mathrm{tx}}^{2}$ is obtained via the ML estimation.

It is noted that [12], [49], [52] investigated parameter estimation for a single RX only. Different from these studies, [50], [51] investigated parameter estimation via two fully-absorbing RXs in a 1D environment. In [50], the authors applied the transfer function to estimate the diffusion coefficient of molecules. Compared to the previous studies, [51] considered the existence of the external additive noise in the parameter estimation process. To reduce the impact of the external additive noise, [51] proposed a novel estimation scheme - difference estimation (DE) - to estimate the unknown parameter based on the difference between received signals at two RXs. According to the CIR derived between the TX and each fully-absorbing RX in [60, eq. (8)], [51] derived the CRLB on the variance of the unknown parameter. By assuming that each observation 
TABLE II

PARAMETER ESTIMATION SCHEMES FOR MC SySTEMS

\begin{tabular}{|c|c|c|c|c|c|c|c|}
\hline Name & $\begin{array}{l}\text { Estimated } \\
\text { Parameter }\end{array}$ & Reference & Method & Noise & TX Waveform & RX Type & Environment \\
\hline \multirow{5}{*}{$\begin{array}{l}\text { Environmental } \\
\text { Parameters }\end{array}$} & $d, t_{0}, D, v, k, N_{\mathrm{tx}}$ & [12] & ML, Peak-Based & $\mathrm{RD}$ & Impulse & Transparent & $\begin{array}{c}\text { 3D, Flow, } \\
\text { Molecules } \\
\text { Degradation }\end{array}$ \\
\hline & $N_{\mathrm{tx}}$ & 449 & HCRLB & $\mathrm{RD}, \mathrm{ISI}$ & Impulse & Transparent & $3 \mathrm{D}$ \\
\hline & $\mathrm{D}$ & [50] & Transfer Function & $\mathrm{RD}$ & Continuous & $\begin{array}{c}\text { Two } \\
\text { Fully-absorbing }\end{array}$ & $1 \mathrm{D}$ \\
\hline & $d, v, k$ & [51] & $\begin{array}{l}\text { Method of } \\
\text { Moments }\end{array}$ & RD, External & Continuous & $\begin{array}{c}\text { Two } \\
\text { Fully-absorbing }\end{array}$ & $\begin{array}{c}\text { 1D, Flow, } \\
\text { Molecules } \\
\text { Degradation }\end{array}$ \\
\hline & $\sigma_{\mathrm{tx}}^{2}$ & [52] & ML & RD & Impulse & Transparent & $1 \mathrm{D}, 2 \mathrm{D}, 3 \mathrm{D}$ \\
\hline \multirow{5}{*}{$\begin{array}{c}\text { Synchronization } \\
\text {-Related Parameters }\end{array}$} & \multirow{3}{*}{ Clock Offset } & [53], โ54] & Two-Way, ML & $\mathrm{RD}$ & Impulse & Transparent & 1D \\
\hline & & 55 & One-Way, ML & $\mathrm{RD}$ & Impulse & Transparent & 1D, Flow \\
\hline & & [56] & Least Square & $\mathrm{RD}$ & $\begin{array}{l}\text { Diffusive, } \\
\text { Impulse }\end{array}$ & $\begin{array}{c}\text { Diffusive, } \\
\text { Fully-absorbing }\end{array}$ & $3 \mathrm{D}$ \\
\hline & \multirow[t]{2}{*}{$t_{\mathrm{S}}(\varepsilon)$} & 57 & $\begin{array}{l}\text { ML, Linear Filter, } \\
\text { Peak Observation, } \\
\text { Threshold-Trigger }\end{array}$ & $\begin{array}{c}\text { External, ISI, } \\
\text { RD }\end{array}$ & Impulse & Reactive & $3 \mathrm{D}$ \\
\hline & & 758 & Faster Molecules & ISI, RD & Impulse & Fully-absorbing & $3 \mathrm{D}$ \\
\hline SNR & SNR & 59 & ML & ISI, RD & Impulse & Transparent & $3 \mathrm{D}$ \\
\hline
\end{tabular}

of the received signal is independent and follows a Poisson distribution, $p(\mathbf{x} ; \theta)$ was obtained as the joint PMF for the difference of received signals at two RXs. In addition, [51] applied the method of moments [29, Ch.9] to estimate the unknown parameter.

\section{B. Synchronization-Related Parameters}

In this subsection, we review the studies on the estimation of synchronization-related parameters that include the clock offset between the TX and the RX, and the start time of each symbol interval. The clock offset describes a time difference between the TX and the RX. In a nanonetwork system, different nanomachines work based on their own clocks. Thus, estimating the clock offset is crucial to establish a reliable communication link between the synchronized TX and RX.

In [53], [54], Lin et al. estimated the clock offset between two transceivers, denoted by $T$ and $R$, respectively, via proposing a two-way message exchange mechanism. In the $\varepsilon$ th round of the message exchange, $T$ sends molecules at time $T_{1, \varepsilon}$, and $R$ receives the message at time $T_{2, \varepsilon} . R$ then sends a feedback signal at time $T_{3, \varepsilon}$, and $T$ receives the signal at time $T_{4, \varepsilon}$. After $\alpha$ rounds of message exchange, $T$ obtains a set of time instants $\left\{T_{1, \varepsilon}, T_{2, \varepsilon}, T_{3, \varepsilon}, T_{4, \varepsilon}\right\}_{\varepsilon=1}^{\alpha}$. By assuming that the propagation delay follows an inverse Gaussian distribution and Gaussian distribution in [53] and [54], respectively, the joint PDF of the molecular propagation delay for the $\alpha$ round message exchange can be obtained. Based on the PDF, the clock offset is estimated via the ML estimation. After that, $R$ can be synchronized to $T$. Moreover, [55] proposed a one-way clock offset estimation method due to the fact that two-way estimation has a high demand for transceivers and is time-consuming as aforementioned in Section IV-A3. Based on the joint PDF of the molecular propagation delay for multiple transmissions from the TX, the clock offset is estimated by the ML estimation. Furthermore, [56] considered clock offset estimation when both TX and RX are diffusive mobile. After molecules are released from the TX, the RX counts the number of arrived molecules for $M$ times. The authors estimated clock offset by using the least square method that finds the clock offset to minimize the sum of differences between the mean of the CIR over the varying distance and $M$ observations.

The estimation of clock offset is adequate to achieve synchronization only if the clock offset is fixed. To overcome this issue, [57], [58] considered the estimation of the start time of the $\eta$ th symbol interval, denoted by $t_{\mathrm{s}}[\eta]$. In [57], the authors first proposed the ML estimation scheme to estimate $t_{\mathrm{s}}[\eta]$. By considering that each observation within this interval follows a Poisson distribution, $t_{\mathrm{s}}[\eta]$ is estimated based on the joint PDF of multiple observations. Due to the high complexity of the ML estimation, the authors then proposed three suboptimal low-complexity estimation schemes. The first suboptimal estimation scheme is a linear filter-based scheme that finds $t_{\mathrm{s}}[\eta]$ to maximize the expected mean of multiple observations. The second one is the peak observation-based scheme that estimates $t_{\mathrm{s}}[\eta]$ based on the peak observation at the RX. The third one is the threshold-trigger scheme that determines $t_{\mathrm{s}}[\eta]$ when the observation is larger than a predefined threshold. Notably, [57] considered the impact of the external additive noise and ISI on these estimation schemes. Moreover, [58] considered the transmission of molecules with a faster diffusion coefficient than information molecules to realize synchronization, where $t_{\mathrm{s}}[\eta]$ is estimated as the time when the peak concentration of faster molecules is detected at the RX.

\section{C. $S N R$}

In [59], Tiwari et al. estimated the SNR in MC system, which considered the noise induced by the ISI. In [59], the SNR was defined as

$$
\mathrm{SNR}=\frac{P_{\mathrm{s}}}{P_{\mathrm{n}}},
$$

where $P_{\mathrm{s}}$ represents the power of the intended received signal at the transparent RX and $P_{\mathrm{n}}$ represents the power of noise 
TABLE III

CIR ESTIMATION SCHEMES FOR MC SYSTEMS

\begin{tabular}{|c|c|c|c|c|c|c|}
\hline Name & References & Method & Performance & Computational Complexity & Noise & TX Waveform \\
\hline \multirow{3}{*}{$\begin{array}{c}\text { Pilot-Based } \\
\text { CIR Estimation }\end{array}$} & {$[15]$} & ML & \multirow{5}{*}{$\begin{array}{c}\mathrm{EM}>\mathrm{DD}>\mathrm{ML} \\
>\mathrm{LSSE}(\mathrm{LO})\end{array}$} & \multirow{5}{*}{$\begin{array}{c}\mathrm{EM}<\mathrm{DD}<\mathrm{ML} \\
<\mathrm{LSSE}(\mathrm{LO})\end{array}$} & RD,ISI & Impulse \\
\hline & 15,61 & LSSE & & & RD, ISI & Impulse \\
\hline & - 16$]$ & ML,LSSE & & & RD,ISI, ILI & Impulse \\
\hline \multirow{2}{*}{$\begin{array}{c}\text { Semi-Blind } \\
\text { CIR Estimation }\end{array}$} & 62 & EM,DD & & & RD, ISI & Impulse \\
\hline & 63 & LO & & & RD,ISI & Impulse \\
\hline $\begin{array}{c}\text { CIR Estimation } \\
\text { Used at RX Design }\end{array}$ & 64 & $\begin{array}{c}\text { MSE, } \\
\text { Steepest-Descent Algorithm }\end{array}$ & N/A & N/A & RD,ISI & Impulse \\
\hline
\end{tabular}

due to the ISI. The power in MC can be interpreted as the square number of molecules. Thus, the SNR is a function of the number of emitted molecules $N_{\mathrm{tx}}$ and the variance of the noise, denoted by $\sigma_{\mathrm{n}}^{2}$, where the noise is regarded as a Gaussian RV. According to the invariance property of the ML estimation, estimating a function with multiple unknown parameters is equivalent to estimating individual unknown parameters [29]. Therefore, the SNR is estimated by using the ML estimation of $N_{\mathrm{tx}}$ and $\sigma_{\mathrm{n}}^{2}$ based on the joint PDF of the received signals at the RX. In addition, the CRLB was derived when $\boldsymbol{\theta}=\left[N_{\mathrm{tx}}^{2}, \sigma_{\mathrm{n}}^{2}\right]$.

\section{Vi. Channel Estimation}

In this section, we first present the CIR estimation problem and then review different CIR estimation schemes. We also summarize different CIR estimation studies in Table III.

\section{A. Problem Formulation}

We consider an MC system as shown in Fig. 1 At the beginning of each symbol interval, the TX releases $N_{\text {tx }}$ molecules if the transmitted symbol is " 1 ", but does not release any molecule if the transmitted symbol is "0". Taking into account the effect of ISI, we assume the input-output relationship of the MC system as

$$
z[q]=\sum_{u=1}^{U} c_{u}[q]+c_{\mathrm{n}}[q],
$$

where $z[q]$ is the number of molecules detected at the RX in symbol interval $q, U$ is the number of memory taps of the channel, and $c_{u}[q]$ is the number of molecules observed at the $\mathrm{RX}$ in symbol interval $u$, due to the release of $b[q-u+1] N_{\mathrm{tx}}$ molecules by the TX in symbol interval $q-u+1$, where $b[q] \in[0,1]$ is the transmitted symbol in symbol interval $q$. Therefore, $c_{u}[q]$ can be well approximated by a Poisson RV with the mean of $\bar{c}_{u} b[q-u+1]$. Moreover, $c_{\mathrm{n}}[q]$ is the number of external additive noise molecules detected by the RX in the symbol interval $q$. Let $\mathbf{b}=[b[1], b[2], \ldots, b[Q]]^{T}$ be a training sequence of length $Q$.

For convenience of notation, we define $\mathbf{z}=[z[U], z[U+$ $1], \ldots, z[Q]]^{T}, \overline{\mathbf{c}}=\left[\bar{c}_{1}, \bar{c}_{2}, \ldots, \bar{c}_{U}, \bar{c}_{\mathrm{n}}\right]^{T}$ is the CIR of the channel, and $f_{\mathbf{z}}(\mathbf{z} \mid \overline{\mathbf{c}}, \mathbf{b})$ is the PDF of the observation $\mathbf{z}$ conditioned on a given channel $\overline{\mathbf{c}}$ and a given training sequence b. The goal of channel estimation is to estimate $\overline{\mathbf{c}}$ based on the vector of random observations $\mathbf{z}$.

\section{B. Pilot-Based CIR Estimation}

In this subsection, we present the pilot-based CIR estimation scheme studied in $[15]^{2}$, where the transmission of a known training sequence of pilots is required for the estimation and calculation of the corresponding CRLB.

1) ML Estimation: The ML CIR estimation scheme aims to find the CIR that maximizes the likelihood of observation vector $\mathbf{z}$ [65]. In particular, the ML estimation is given by

$$
\hat{\overline{\mathbf{c}}}^{\mathrm{ML}}=\underset{\mathbf{\mathbf { c }} \geq \mathbf{0}}{\operatorname{argmax}} f_{\mathbf{z}}(\mathbf{z} \mid \overline{\mathbf{c}}, \mathbf{b}) .
$$

We assume that $z[q]$ is a Poisson RV with the mean of $\bar{z}[q]=$ $\bar{c}_{\mathrm{n}}+\sum_{u=1}^{U} \bar{c}_{u} b[q-u+1]=\overline{\mathbf{c}}^{T} \mathbf{b}_{q}$ and $\mathbf{b}_{q}=[b[q], b[q-$ $1], \ldots, b[q-U+1], 1]^{T}$. Under this assumption, $f_{\mathbf{z}}(\mathbf{z} \mid \overline{\mathbf{c}}, \mathbf{b})$ is given by

$$
f_{\mathbf{z}}(\mathbf{z} \mid \overline{\mathbf{c}}, \mathbf{b})=\prod_{q=U}^{Q} \frac{\left(\overline{\mathbf{c}}^{T} \mathbf{b}_{q}\right)^{z[q]}}{z[q] !} \exp \left(-\overline{\mathbf{c}}^{T} \mathbf{b}_{q}\right) .
$$

We note that [15] solved the ML estimation of the CIR given in 31 by using Algorithm 1, where the following non-linear system of equations is solved 3 for different $\mathcal{A}_{w}$

$$
\sum_{q=L}^{Q}\left[\frac{z[q]}{\left(\overline{\mathbf{c}}^{\mathcal{A}_{w}}\right)^{T} \mathbf{b}_{q}^{\mathcal{A}_{w}}}-1\right] \mathbf{b}_{q}^{\mathcal{A}_{w}}=\mathbf{0},
$$

where $\mathcal{A}=\left\{\mathcal{A}_{1}, \mathcal{A}_{2}, \cdots, \mathcal{A}_{W}\right\}$ denotes a set which contains all possible $W=2^{U+1}-1$ subsets of the set $\mathcal{F}=$ $\{1,2, \cdots, U, \mathrm{n}\}$, except for the empty set. Here, $\mathcal{A}_{w}$ denotes the $w$-th subset of $\mathcal{A}, w=1,2, \cdots, W$. Moreover, let $\overline{\mathbf{c}}^{\mathcal{A}_{w}}$ and $\mathbf{b}_{q}^{\mathcal{A}_{w}}$ denote the reduced-dimension versions of $\overline{\mathbf{c}}$ and $\mathbf{b}_{q}$, respectively, which only contain those elements of $\overline{\mathbf{c}}$ and $\mathbf{b}_{q}$ whose indices are the elements of $\mathcal{A}_{w}$, respectively.

2) Least Sum of Squared Errors (LSSE) CIR Estimation: The LSSE CIR estimation scheme aims to choose $\overline{\mathbf{c}}$ that minimizes the sum of the squared errors for the observation vector $\mathbf{z}$. Here, the error vector is defined as $\boldsymbol{\epsilon}=\mathbf{z}-\mathbb{E}\{\mathbf{z}\}=\mathbf{z}-\mathbf{B} \overline{\mathbf{c}}$ where $\mathbf{B}=\left[\mathbf{b}_{U}, \mathbf{b}_{U+1}, \ldots, \mathbf{b}_{Q}\right]^{T}$. In particular, the LSSE CIR estimation can be written as

$$
\hat{\overline{\mathbf{c}}}^{\text {LSSE }}=\underset{\overline{\mathbf{c}} \geq \mathbf{0}}{\operatorname{argmin}}\|\boldsymbol{\epsilon}\|^{2} .
$$

\footnotetext{
${ }^{2}$ We clarify that both [15] and [16] are technical papers rather than comprehensive surveys on MC channel estimation.

${ }^{3} \mathrm{~A}$ system of nonlinear equations can be solved by using mathematical software packages, e.g., Mathematica.
} 


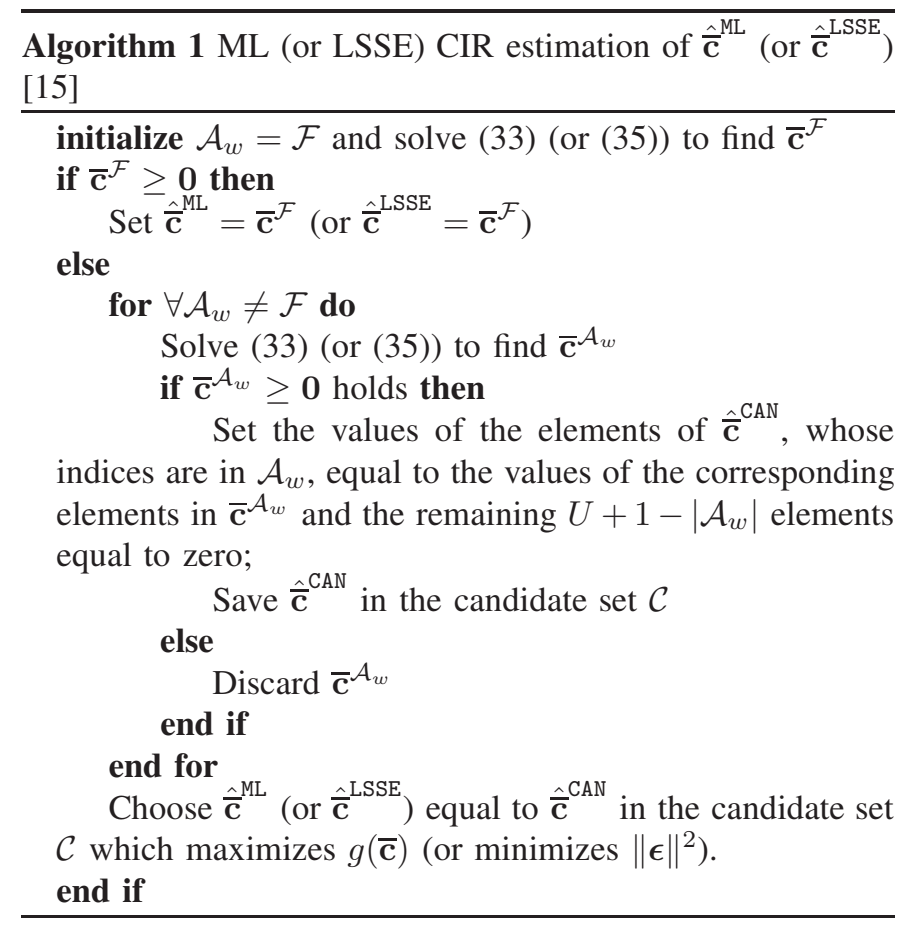

It is noted that [15] also obtained the LSSE estimation of the CIR by solving (34). The solution is given by Algorithm 1 where for a given set $\mathcal{A}_{w}, \overline{\mathbf{c}}^{\mathcal{A}_{w}}$ is obtained as

$$
\overline{\mathbf{c}}^{\mathcal{A}_{w}}=\left(\left(\mathbf{B}^{\mathcal{A}_{w}}\right)^{T} \mathbf{B}^{\mathcal{A}_{w}}\right)^{-1}\left(\mathbf{B}^{\mathcal{A}_{w}}\right)^{T} \mathbf{z} .
$$

We also note that [61] designed a communication protocol that estimates the CIR based on the least square method, similar to the LSSE CIR estimation in [15].

3) CRLB: With the estimation error vector defined as $\mathbf{e}=$ $\overline{\mathbf{c}}-\hat{\overline{\mathbf{c}}}$, the classical CRLB for the deterministic $\overline{\mathbf{c}}$ provides the following lower bound on the sum of the expected square errors [15]

$$
\mathbb{E}\left\{\|\mathbf{e}\|^{2}\right\} \geq \operatorname{tr}\left\{\mathbf{I}^{-1}(\overline{\mathbf{c}})\right\}=\operatorname{tr}\left\{\left[\sum_{q=U}^{Q} \frac{\mathbf{b}_{q} \mathbf{b}_{q}^{T}}{\overline{\mathbf{c}}^{T} \mathbf{b}_{q}}\right]^{-1}\right\},
$$

where $\operatorname{tr}\{\cdot\}$ denotes the trace of a matrix. While [15] derived the ML and LSSE estimation schemes of the CIR and the CRLB for a single-input single-output channel, [16] extended these results to a diffusive MIMO system, by incorporating the ILI.

\section{Semi-Blind CIR Estimation}

In this subsection, we present semi-blind CIR estimation schemes where the transmission of $Q$ pilot symbols is followed by the transmission of $\mathcal{D}$ unknown data symbols, denoted by the vector $\boldsymbol{\beta}=[\beta[1], \beta[2], \ldots, \beta[\mathcal{D}]]^{T}$. The semi-blind CIR estimation schemes incorporate both data-carrying and pilot-carrying observations into the estimation process. This is different from pilot-based estimation which only considers the received pilot-carrying observations in the estimation process but excludes data-carrying observations. Incorporating datacarrying observations into the estimation can significantly

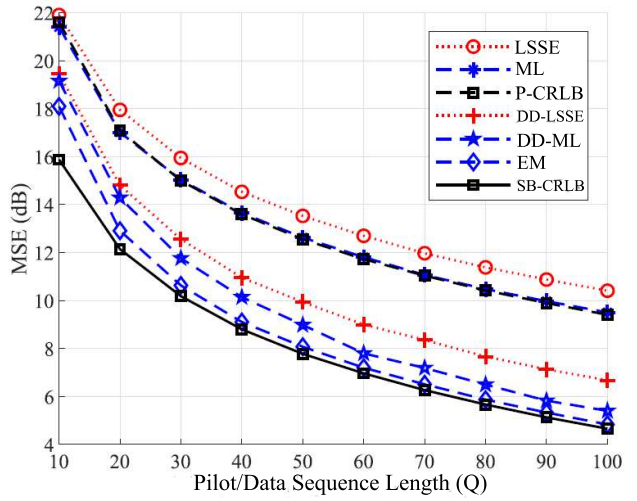

Fig. 6. The MSE of different CIR estimation schemes versus pilot sequence length 62. Fig. 1]. LSSE: least squares, ML: maximum-likelihood, P-CRLB: Pilot-Based CRLB, DD-ML: decision-directed ML, DD-LS: decision-directed LS, SB-CRLB: semi-blind CRLB.

enhance the estimation accuracy and/or improve the data rate [62].

An expectation maximization (EM)-based estimation scheme was first proposed in [62]. The data vector $\boldsymbol{\beta}$ constitutes hidden information at the RX. Beginning with the initial guess, the EM estimation scheme alternates between obtaining the conditional expectation of the complete-data log-likelihood and maximizing the result with respect to the desired parameters. The $\omega$ th iteration of the EM estimation scheme consists of two steps. The first is the expectation step (E-step) which consists of obtaining the expectation of the complete-data log-likelihood function. This is followed by the maximization step (M-step), in which the posterior probability of the hidden data is maximized to acquire an updated estimate of the CIR. Abdallan et al. in [62] also proposed two semi-blind estimation schemes based on the decision-directed (DD) strategy. The idea of the DD strategy is to use the channel estimate acquired through pilot-based estimation for performing data detection. The detected data is in turn treated as a new set of pilots to perform another cycle of channel estimation [62]. We note that the analytical derivation of the CRLB can be very challenging in semi-blind estimation, because the log-likelihood function of observations becomes complicated when the statistics of data symbols are taken into account. In [62], the authors applied the MonteCarlo method to obtain accurate approximations of the Fisher information matrix $\mathbf{I}^{-1}(\overline{\mathbf{c}})$. The semi-blind CRLB was then obtained by evaluating $\operatorname{tr}\left\{\mathbf{I}^{-1}(\overline{\mathbf{c}})\right\}$.

Darya et al. in [63] proposed a modified version of the decision-directed least-squares (DDLS) estimation scheme proposed in [62]. In [63], the authors named the proposed estimation scheme as the low-overhead (LO) estimation scheme which has reduced complexity compared to the DDLS estimation scheme. The authors in [63] showed that the LO estimation scheme has comparable performance to the pilot-based LS estimation scheme in [15], while achieving a reduction of up to $95 \%$ in the pilot overhead by using a minimal number of pilot symbols. 


\section{Pilot-Based Estimation versus Semi-Blind Estimation}

Based on the simulation results in Fig. 6 (we re-presented Fig. 1 in [62] as Fig. 6 here for convenience), the semi-blind estimation schemes achieve a significantly lower MSE than the existing pilot-based ML and LSSE estimation schemes. Also, the semi-blind estimation schemes can substantially reduce the pilot overhead as compared to the best-performing pilot-based estimation schemes, by more than $60 \%$ for the case of EM and $55 \%-44 \%$ for DD-based estimation. The EM-based semi-blind estimation scheme provides the highest estimation accuracy. The DD-based semi-blind estimation scheme performs almost midway between the pilot-based ML and the EM-based semiblind estimation schemes, but achieves a lower computational cost than the EM-based semi-blind estimation scheme.

\section{E. Channel Estimation Scheme at the RX design}

In this subsection, we present a joint channel and data estimation scheme that was presented in [64], while [15], [16], [62] only focused on channel estimation based on given training sequences. Specifically, [64] presented an estimation scheme which is compatible with different detectors (e.g., the MAP and ML sequence detectors) at the RX to jointly recover the transmitted bits from the molecule observations distorted by both the ISI and noise. This is because that the detectors at the RX require the knowledge of the CIR. In [64], the channel estimation scheme uses a steepest-descent algorithm to recursively estimate the CIR which minimizes the MSE between the actual received sequence and the output of the estimation scheme. The speed of the convergence and the accuracy of the estimation are determined by the value of the step size in the steepest-descent algorithm. Also, due to the use of the steepest-descent algorithm, the channel estimation scheme is able to track slow variations in the CIR.

\section{Future Research Directions}

In this section, we identify and discuss some future research directions for parameter estimation and channel estimation in MC systems. Current studies on estimation have usually considered simple TX and RX protocols and ideal communication channels. These simplifications would lead to inaccuracy when the current estimation schemes are applied into practical MC environments. Moreover, the noise is common in MC systems. Effective methods to mitigate the impact of noise on the estimation performance need to be widely investigated. Based on these, we present some open research problems as follows:

- Imperfect TX: Current studies on estimation schemes have considered an ideal point TX. Compared to realistic scenarios, this ideal TX model does not address the properties of the TX, such as geometry, signaling pathways inside the TX, and chemical reactions during the release process. Some recent studies have proposed imperfect TX models, e.g., an ion channel-based TX in [66] and a membrane fusion-based TX in [67]. Applying these imperfect TX into estimation schemes is an interesting future work.

- Macro-scale estimation: Most current studies have examined the estimation in the micro-scale environment while a few studies have considered macro-scale estimation. As aforementioned, MC also exists in macroscale. Thus, the estimation in the macro-scale environment needs attention. Here, we propose two research directions. The first direction is to perform estimation based on tabletop experiments. Some studies have established the tabletop experiment for macro-scale MC, e.g., [47], [68], [69]. Estimation can be investigated based on these experiments. The second direction is the theoretical analysis of estimation schemes in practical macro-scale environments, e.g., a pipe or river. Compared to traditional theoretical analysis in micro-scale MC, flow modeling is crucial for the analysis in the macro-scale environment. For example, the flow can be modeled as laminar in the pipe and turbulent in the river.

- Noise mitigation: Most studies have included noise, e.g., $\mathrm{RD}$, ISI, and external additive noise, in the estimation process, while only a few of them, e.g., [51], proposed methods to mitigate the impact of noise on estimation. It is noted that [51] only focused on a stable stage of the communication channel, i.e., the expected received signal is constant, when time is large. More general noise mitigation methods should be investigated during the estimation process.

- MIMO estimation: Most of existing studies have focused on the estimation via one TX and one RX. Only a limited number of studies, such as [51], investigated cooperative estimation via two RXs and showed the improved estimation accuracy as compared to the singleRX estimation. Meanwhile, a few studies, such as [70], showed that the detection performance of transmitted symbols is greatly improved by combining the received information at multiple distributed receivers. Based on these studies, the performance enhancement in parameter or channel estimation by combining the received information at multiple receivers has not been thoroughly explored. Moreover, estimation schemes by using the MIMO system have not been investigated. In particular, multiple channels can be adopted to estimate multiple unknown parameters.

- Real-time parameter estimation: The existing studies have focused on one-shot parameter estimation by assuming that the estimated parameters are constant over time, or have focused on the estimation of the initial value of parameters when the estimated parameters keep changing over time. However, in dynamic biological environments, many parameters vary over time, e.g., the distance between the TX and RX changes when the transceivers move. Hence, the real-time estimation of time-varying parameters is a promising research direction.

\section{CONCLUSION}

In this paper, we provided the first comprehensive survey on parameter estimation and channel estimation in MC systems. We summarized three types of noise that can influence the estimation performance and three metrics that can be used to evaluate the performance of an estimation scheme. For 
parameter estimation, we first presented a detailed review on the distance estimation and compare the performance of different estimation schemes via calculating the MSE. We then provided a detailed review on the estimation of other parameters. For channel estimation, we reviewed the pilot-based CIR estimation scheme and the semi-blind CIR estimation scheme. Finally, we discussed some open research problems for parameter estimation and channel estimation. This survey helps $\mathrm{MC}$ researchers to develop an in-depth understanding on the current estimation schemes in MC and serves as a cornerstone for MC researchers to explore more advanced estimation schemes in the future.

\section{REFERENCES}

[1] N. Farsad, H. B. Yilmaz, A. Eckford, C.-B. Chae, and W. Guo, "A comprehensive survey of recent advancements in molecular communication," IEEE Commun. Surv. Tut., vol. 18, no. 3, pp. 1887-1919, 3rd Quarter 2016.

[2] U. A. Chude-Okonkwo, R. Malekian, B. T. Maharaj, and A. V. Vasilakos, "Molecular communication and nanonetwork for targeted drug delivery: A survey," IEEE Commun. Surv. Tut., vol. 19, no. 4, pp. 3046-3096, 4th Quarter 2017.

[3] V. Jamali, A. Ahmadzadeh, W. Wicke, A. Noel, and R. Schober, "Channel modeling for diffusive molecular communication-a tutorial review," Proc. IEEE, vol. 107, no. 7, pp. 1256-1301, Jul. 2019.

[4] M. Kuscu, E. Dinc, B. A. Bilgin, H. Ramezani, and O. B. Akan, "Transmitter and receiver architectures for molecular communications: A survey on physical design with modulation, coding, and detection techniques," Proc. IEEE, vol. 107, no. 7, pp. 1302-1341, Jul. 2019.

[5] T. Nakano, Y. Okaie, S. Kobayashi, T. Hara, Y. Hiraoka, and T. Haraguchi, "Methods and applications of mobile molecular communication," Proc. IEEE, vol. 107, no. 7, pp. 1442-1456, Jul. 2019.

[6] M. J. Moore and T. Nakano, "Addressing by beacon distances using molecular communication," Nano Commun. Netw., vol. 2, no. 2-3, pp. 161-173, 2011.

[7] F. Tostevin, P. R. Ten Wolde, and M. Howard, "Fundamental limits to position determination by concentration gradients," PLoS Computat. Biol., vol. 3, no. 4, pp. 763-771, Apr. 2007.

[8] C.-H. Ho, "White blood cell and platelet counts could affect whole blood viscosity," J. Chin. Med. Assoc., vol. 67, no. 8, pp. 394-397, Aug. 2004.

[9] R. Chang, Physical Chemistry for the Biosciences. Sausalito, CA, USA: Univ. Science Books, 2005.

[10] M. J. Moore, T. Nakano, A. Enomoto, and T. Suda, "Measuring distance from single spike feedback signals in molecular communication," IEEE Trans. Signal Process., vol. 60, no. 7, pp. 3576-3587, Jul. 2012.

[11] X. Wang, M. D. Higgins, and M. S. Leeson, "Distance estimation schemes for diffusion based molecular communication systems," IEEE Commun. Lett., vol. 19, no. 3, pp. 399-402, Mar. 2015.

[12] A. Noel, K. C. Cheung, and R. Schober, "Joint channel parameter estimation via diffusive molecular communication," IEEE Trans. Mol. Biol. Multi-Scale Commun., vol. 1, no. 1, pp. 4-17, Mar. 2015.

[13] _ - "Optimal receiver design for diffusive molecular communication with flow and additive noise," IEEE Trans. Nanobiosci., vol. 13, no. 3, pp. 350-362, Sep. 2014.

[14] M. U. Mahfuz, D. Makrakis, and H. T. Mouftah, "A comprehensive study of sampling-based optimum signal detection in concentration-encoded molecular communication," IEEE Trans. Nanobiosci., vol. 13, no. 3, pp. 208-222, Sep. 2014.

[15] V. Jamali, A. Ahmadzadeh, C. Jardin, H. Sticht, and R. Schober, "Channel estimation for diffusive molecular communications," IEEE Trans. Commun., vol. 64, no. 10, pp. 4238-4252, Oct. 2016.

[16] S. M. R. Rouzegar and U. Spagnolini, "Diffusive MIMO molecular communications: Channel estimation, equalization, and detection," IEEE Trans. Commun., vol. 67, no. 7, pp. 4872-4884, Jul. 2019.

[17] A. Noel, K. C. Cheung, and R. Schober, "Improving receiver performance of diffusive molecular communication with enzymes," IEEE Trans. NanoBiosci., vol. 13, no. 1, pp. 31-43, Mar. 2014.

[18] _ - "Using dimensional analysis to assess scalability and accuracy in molecular communication," in Proc. IEEE ICC Workshops 2013, Budapest, Hungary, Jun. 2013, pp. 818-823.
[19] _ - "Bounds on distance estimation via diffusive molecular communication," in Proc. IEEE Globecom 2014, Austin, TX, USA, Dec. 2014, pp. 2813-2819.

[20] P. Cuatrecasas, "Membrane receptors," Апnи. Rev. Biochem., vol. 43, no. 1, pp. 169-214, Jul. 1974.

[21] H. B. Yilmaz, A. C. Heren, T. Tugcu, and C.-B. Chae, "Threedimensional channel characteristics for molecular communications with an absorbing receiver," IEEE Commun. Lett., vol. 18, no. 6, pp. 929-932, Jun. 2014.

[22] A. Ahmadzadeh, H. Arjmandi, A. Burkovski, and R. Schober, "Comprehensive reactive receiver modeling for diffusive molecular communication systems: Reversible binding, molecule degradation, and finite number of receptors," IEEE Trans. Nanobiosci., vol. 15, no. 7, pp. 713727, Oct. 2016.

[23] M. Pierobon and I. F. Akyildiz, "Diffusion-based noise analysis for molecular communication in nanonetworks," IEEE Trans. Signal Process., vol. 59, no. 6, pp. 2532-2547, Jun. 2011.

[24] A. S. Ladokhin, W. C. Wimley, and S. H. White, "Leakage of membrane vesicle contents: Determination of mechanism using fluorescence requenching," Biophys. J., vol. 69, no. 5, pp. 1964-1971, Nov. 1995.

[25] B. Alberts, D. Bray, K. Hopkin, A. D. Johnson, J. Lewis, M. Raff, K. Roberts, and P. Walter, Essential Cell Biology, 3rd ed. New York, NY, USA: Garland, 2010.

[26] M. Falk, J. Hüsler, and R.-D. Reiss, Laws of Small Numbers: Extremes and Rare Events. Springer, 2010.

[27] H. Pishro-Nik, Introduction to Probability, Statistics, and Random Processes. Kappa Research, LLC, 2016.

[28] D. Wackerly, W. Mendenhall, and R. L. Scheaffer, Mathematical Statistics with Applications. Pacific Grove, CA, USA: Duxbury, 2002.

[29] S. M. Kay, Fundamentals of Statistical Signal Processing: Estimation Theory. Upper Saddle River, NJ, USA: Prentice-Hall, 1993.

[30] E. L. Lehmann and G. Casella, Theory of Point Estimation. New York, NY: Springer-Verlag, 2006.

[31] M. J. Moore and T. Nakano, "Comparing transmission, propagation, and receiving options for nanomachines to measure distance by molecular communication," in Proc. IEEE ICC 2012, Ottawa, ON, Canada, Jun. 2012, pp. 6132-6136

[32] J.-T. Huang, H.-Y. Lai, Y.-C. Lee, C.-H. Lee, and P.-C. Yeh, "Distance estimation in concentration-based molecular communications," in Proc. IEEE Globecom 2013, Atlanta, GA, USA, Dec. 2013, pp. 2587-2591.

[33] S. Liu, S. Bao, and C. Zhao, "Localization schemes for 2-D molecular communication via diffusion," in Proc. CSPS 2019, Apr. 2019, pp. 749756.

[34] M. Turan, B. C. Akdeniz, M. S. Kuran, H. B. Yilmaz, I. Demirkol, A. E. Pusane, and T. Tugcu, "Transmitter localization in vessel-like diffusive channels using ring-shaped molecular receivers," IEEE Commun. Lett., vol. 22, no. 12, pp. 2511-2514, Dec. 2018.

[35] Z. Luo, L. Lin, Q. Fu, and H. Yan, "An effective distance measurement method for molecular communication systems," in Proc. IEEE SECON Workshops 2018, Hong Kong, Jun. 2018, pp. 1-4.

[36] Y. Sun, M. Ito, and K. Sezaki, "An efficient distance measurement approach in diffusion-based molecular communication based on arrival time difference," in Proc. ACM NanoCom 2017, Washington DC,USA, Sep. 2017, pp. 1-6.

[37] S. Kumar, "Nanomachine localization in a diffusive molecular communication system," IEEE Syst. J., vol. 14, no. 2, pp. 3011-3014, Jun. 2020.

[38] L. Lin, Z. Luo, L. Huang, C. Luo, Q. Wu, and H. Yan, "High-accuracy distance estimation for molecular communication systems via diffusion," Nano Commun. Netw., vol. 19, pp. 47-53, Mar. 2019.

[39] S. Huang, L. Lin, W. Guo, H. Yan, J. Xu, and F. Liu, "Initial distance estimation and signal detection for diffusive mobile molecular communication," IEEE Trans. Nanobiosci., vol. 19, no. 3, pp. 422-433, Jul. 2020.

[40] X. Wang, M. D. Higgins, and M. S. Leeson, "An algorithmic distance estimation scheme for diffusion based molecular communication systems," in Proc. IEEE ICC 2015, London, UK, Jun. 2015, pp. 1134-1139.

[41] Y. Miao, W. Zhang, and X. Bao, "Cooperative source positioning for simo molecular communication via diffusion," in Proc. IEEE ICCT 2019, Xi'an, China, Oct. 2019, pp. 495-499.

[42] F. Gulec and B. Atakan, "Distance estimation methods for a practical macroscale molecular communication system," Nano Commun. Netw., vol. 24, p. 100300, May 2020.

[43] H. Bruus, Theoretical Microfluidics, 1st ed. Oxford, U.K.: Oxford Univ Press, 2007. 
[44] W. Wicke, T. Schwering, A. Ahmadzadeh, V. Jamali, A. Noel, and R. Schober, "Modeling duct flow for molecular communication," in Proc. IEEE Globecom 2018, Abu Dhabi, United Arab Emirates, Dec. 2018, pp. 206-212.

[45] M. Schäfer, W. Wicke, L. Brand, R. Rabenstein, and R. Schober, "Transfer function models for cylindrical mc channels with diffusion and laminar flow," Jul. 2020. [Online]. Available: arXiv:2007.01799

[46] J. J. Moré, "The Levenberg-Marquardt algorithm: Implementation and theory," Numer. Anal., pp. 105-116, 1978

[47] N. Farsad, W. Guo, and A. W. Eckford, "Tabletop molecular communication: Text messages through chemical signals," PloS One, vol. 8, no. 12, p. e82935, Dec. 2013.

[48] S. S. Andrews and D. Bray, "Stochastic simulation of chemical reactions with spatial resolution and single molecule detail," Phys. Biol., vol. 1, no. 3, p. 137, Aug. 2004.

[49] A. Sadeghi, S. Ghavami, and G. B. Giannakis, "Performance bounds of estimators in molecular communications under structural constraints," in Proc. ACM NanoCom 2017, Sep. 2017, pp. 1-6.

[50] M. Schäfer, A. Ruderer, and R. Rabenstein, "An eigenfunction approach to parameter estimation for 1D diffusion problems," in Proc. ECC 2019, Jun. 2019, pp. 3784-3789.

[51] X. Huang, Y. Fang, A. Noel, and N. Yang, "Parameter estimation in a noisy $1 \mathrm{D}$ environment via two absorbing receivers," in Proc. IEEE Globecom 2020, Taipei, Taiwan, Dec. 2020.

[52] L.-S. Meng, P.-C. Yeh, K.-C. Chen, and I. F. Akyildiz, "On receiver design for diffusion-based molecular communication," IEEE Trans. Signal Process., vol. 62, no. 22, pp. 6032-6044, Nov. 2014.

[53] L. Lin, C. Yang, M. Ma, and S. Ma, "Diffusion-based clock synchronization for molecular communication under inverse Gaussian distribution," IEEE Sens. J., vol. 15, no. 9, pp. 4866-4874, Sep. 2015.

[54] L. Lin, C. Yang, M. Ma, S. Ma, and H. Yan, "A clock synchronization method for molecular nanomachines in bionanosensor networks," IEEE Sens. J., vol. 16, no. 19, pp. 7194-7203, Oct. 2016.

[55] L. Lin, J. Zhang, M. Ma, and H. Yan, "Time synchronization for molecular communication with drift," IEEE Commun. Lett., vol. 21, no. 3, pp. 476-479, Mar. 2017.

[56] L. Huang, L. Lin, F. Liu, and H. Yan, "Clock synchronization for mobile molecular communication systems," IEEE Trans. NanoBiosci., Dec. 2020.

[57] V. Jamali, A. Ahmadzadeh, and R. Schober, "Symbol synchronization for diffusion-based molecular communications," IEEE Trans. NanoBiosci., vol. 16, no. 8, pp. 873-887, Dec. 2017.

[58] M. Mukherjee, H. B. Yilmaz, B. B. Bhowmik, J. Lloret, and Y. Lv, "Synchronization for diffusion-based molecular communication systems via faster molecules," in Proc. IEEE ICC 2019, Shanghai, China, May 2019 , pp. 1-5

[59] S. K. Tiwari and P. K. Upadhyay, "Maximum likelihood estimation of SNR for diffusion-based molecular communication," IEEE Wireless Commun. Lett., vol. 5, no. 3, pp. 320-323, Jun. 2016.

[60] X. Huang, Y. Fang, A. Noel, and N. Yang, "Channel characterization for 1-D molecular communication with two absorbing receivers," IEEE Commun. Lett., vol. 24, no. 6, pp. 1150-1154, Jun. 2020.

[61] J. Wang, D. Hu, C. Shetty, and H. Hassanieh, "Understanding and embracing the complexities of the molecular communication channel in liquids," in Proc. ACM MobiCom 2020, London, United Kingdom, Sep. 2020, pp. 1-15.

[62] S. Abdallah and A. M. Darya, "Semi-blind channel estimation for diffusive molecular communication," IEEE Commun. Lett., vol. 24, no. 11, pp. 2503-2507, Nov. 2020.

[63] A. M. Darya and S. Abdallah, "Low-overhead channel estimation for diffusive molecular communication," in Proc. IEEE ICSPIS 2020, Dubai, United Arab Emirates, Nov. 2020, pp. 1-4.

[64] D. Kilinc and O. B. Akan, "Receiver design for molecular communication," IEEE J. Sel. Areas Commun., vol. 31, no. 12, pp. 705-714, Dec. 2013.

[65] H. S. S. A. Gelman, J. B. Carlin and D. B. Rubin, Bayesian Data Analysis. New York, NY, USA: Taylor \& Francis, 2014.

[66] H. Arjmandi, A. Ahmadzadeh, R. Schober, and M. N. Kenari, "Ion channel based bio-synthetic modulator for diffusive molecular communication," IEEE Trans. Nanobiosci., vol. 15, no. 5, pp. 418-432, Jul. 2016.

[67] X. Huang, Y. Fang, A. Noel, and N. Yang, "Membrane fusion-based transmitter design for molecular communication systems," in Proc. IEEE ICC 2021, Montreal, Canada, Jun. 2021.

[68] D. T. Mcguiness, S. Giannoukos, S. Taylor, and A. Marshall, "Experimental and analytical analysis of macro-scale molecular communications within closed boundaries," IEEE Trans. Mol. Biol. Multi-Scale Commun., vol. 5, no. 1, pp. 44-55, Oct. 2019.

[69] L. Grebenstein, J. Kirchner, W. Wicke, A. Ahmadzadeh, V. Jamali, G. Fischer, R. Weigel, A. Burkovski, and R. Schober, "A molecular communication testbed based on proton pumping bacteria: Methods and data," IEEE Trans. Mol. Biol. Multi-Scale Commun., vol. 5, no. 1, pp. 56-62, Oct. 2019.

[70] Y. Fang, A. Noel, N. Yang, A. W. Eckford, and R. A. Kennedy, "Symbolby-symbol maximum likelihood detection for cooperative molecular communication," IEEE Trans. Commun., vol. 67, no. 7, pp. 4885-4899, Jul. 2019. 